\title{
Sums of products of the degenerate Euler numbers
}

Ming $\mathrm{Wu}^{1}$ and Hao $\mathrm{Pan}^{2 *}$

\section{Abstract}

The paper focuses on the degenerate Euler numbers, the degenerate Euler polynomials and the degenerate Bernoulli polynomials. By adopting the method of recurrences, two explicit expressions have been established for sums of products of the degenerate Euler polynomials and the degenerate Bernoulli polynomials. As a special case of the degenerate Euler polynomials, an expression can be obtained for $\sum_{\substack{1 \\ 1_{1}+j_{2}+\cdots+j_{N}=n}}\left(\begin{array}{c}n \\ j_{1} j_{2} \ldots \ldots j_{2} \cdots j_{N}\end{array}\right) \varepsilon_{j_{1}}(\lambda) \varepsilon_{j_{2}}(\lambda) \cdots \varepsilon_{j_{N}}(\lambda)$

MSC: Primary 11B68; secondary 11B65; 11B73

Keywords: degenerate Euler number; degenerate Euler polynomial; degenerate Bernoulli polynomial

\section{Introduction}

The Bernoulli numbers $B_{n}$ are defined by

$$
\frac{t}{e^{t}-1}=\sum_{n=0}^{\infty} B_{n} \frac{t^{n}}{n !}
$$

The study of the Bernoulli numbers has a very long history. In fact, Euler had found that

$$
\sum_{k=0}^{n}\left(\begin{array}{l}
n \\
k
\end{array}\right) B_{k} B_{n-k}=-(n-1) B_{n}-n B_{n-1}
$$

for any $n \geq 1$.

In 1996, Dilcher [1] generalized (1.1) to the sums of products of $N$ Bernoulli numbers:

$$
\begin{aligned}
& \sum_{\substack{j_{1}+j_{2}+\cdots+j_{N}=n \\
j_{1}, j_{2}, \ldots, j_{N} \geq 0}}\left(\begin{array}{c}
n \\
j_{1}, j_{2}, \ldots, j_{N}
\end{array}\right) B_{j_{1}} B_{j_{2}} \cdots B_{j_{N}} \\
& =N\left(\begin{array}{c}
n \\
N
\end{array}\right) \sum_{j=0}^{N-1}(-1)^{N-1-j} s(N, N-j) \cdot \frac{B_{n-j}}{n-j},
\end{aligned}
$$

where $\left(\underset{j_{1}, j_{2}, \ldots, j_{N}}{n}\right)$ are the multinomial coefficients defined by

$$
\left(\begin{array}{c}
n \\
j_{1}, j_{2}, \ldots, j_{N}
\end{array}\right)=\frac{n !}{j_{1} ! j_{2} ! \cdots j_{N} !}
$$

๑2014 Wu and Pan; licensee Springer. This is an Open Access article distributed under the terms of the Creative Commons Attribution License (http://creativecommons.org/licenses/by/2.0), which permits unrestricted use, distribution, and reproduction in any medium, provided the original work is properly cited. 
and $s(n, k)$ are the Stirling numbers of the first kind (see, e.g., [2]). Furthermore, Dilcher also extended (1.2) to the Bernoulli polynomials.

On the other hand, in [3, 4], Carlitz defined the degenerate Bernoulli numbers $\beta_{n}(\lambda)$ as

$$
\frac{t}{(1+\lambda t)^{1 / \lambda}-1}=\sum_{n=0}^{\infty} \beta_{n}(\lambda) \frac{t^{n}}{n !}
$$

Carlitz showed that $\beta_{n}(\lambda)$ is a polynomial in $\lambda$. And the explicit formula for $\beta_{n}(\lambda)$ was obtained by Howard [5]. Note that $(1+\lambda t)^{1 / \lambda}$ tends to $e^{t}$ as $\lambda \rightarrow 0$. So we have $\beta_{n}(0)=B_{n}$. Furthermore, we know that $\lambda^{-m} \beta_{m}(\lambda)$ tends to $m ! b_{n}$ as $\lambda \rightarrow+\infty$, where $b_{n}$ is the Bernoulli number of the second kind given by

$$
\frac{t}{\log (1+t)}=\sum_{n=0}^{\infty} b_{n} t^{n}
$$

For more number-theoretical properties of the degenerate Bernoulli numbers, the readers may refer to $[6,7]$ and $[8]$.

The analogues of (1.1) and (1.2) for $b_{n}$ have been given in [9] and [10]. Moreover, in [11], Zhang and Yang obtained a generalization of (1.2) for $\beta_{n}(\lambda)$ :

$$
\begin{array}{r}
\sum_{\substack{j_{1}+j_{2}+\cdots+j_{N}=n \\
j_{1}, j_{2}, \ldots, j_{N} \geq 0}}\left(\begin{array}{c}
n \\
j_{1}, j_{2}, \ldots, j_{N}
\end{array}\right) \beta_{j_{1}}(\lambda) \beta_{j_{2}}(\lambda) \cdots \beta_{j_{N}}(\lambda) \\
=N\left(\begin{array}{c}
n \\
N
\end{array}\right) \sum_{k=0}^{N-1}(-1)^{N-1-k} \sigma_{N-1, k}(\lambda, n) \cdot \frac{\beta_{n-k}(\lambda)}{n-k},
\end{array}
$$

where

$$
\sigma_{N-1, k}(\lambda, n)=\sum_{1 \leq i_{k}<i_{k-1}<\cdots<i_{2}<i_{1} \leq N-1} \prod_{j=1}^{k}\left((\lambda-1) i_{j}-(n-j) \lambda\right) .
$$

The Euler numbers $E_{n}$ are another important kind of numbers, which are defined by

$$
\frac{2}{e^{t}+e^{-t}}=\sum_{n=0}^{\infty} E_{n} \frac{t^{n}}{n !}
$$

The Euler numbers have many similar properties as the Bernoulli numbers. For example, in the same paper, Dilcher also proved that

$$
\begin{aligned}
& \sum_{\substack{j_{1}+j_{2}+\cdots+j_{N}=n \\
j_{1}, j_{2}, \ldots, j_{N} \geq 0}}\left(\begin{array}{c}
2 n \\
2 j_{1}, 2 j_{2}, \ldots, 2 j_{N}
\end{array}\right) E_{2 j_{1}} E_{2 j_{2}} \cdots E_{2 j_{N}} \\
& =\frac{2^{2 n+N-1}}{(N-1) !} \sum_{j=0}^{[(N-1) / 2]}\left(\sum_{k=0}^{2 j}\left(\begin{array}{c}
N-1-k \\
2 j-k
\end{array}\right) s(N, N-k) \cdot \frac{N^{2 j-k}}{2^{2 j-k}}\right) E_{2 n+N-1-2 j}\left(\frac{N}{2}\right),
\end{aligned}
$$


where $2 n>N$ and the Euler polynomials $E_{n}(x)$ are given by

$$
\frac{2 e^{x t}}{e^{t}+1}=\sum_{n=0}^{\infty} E_{n}(x) \frac{t^{n}}{n !}
$$

In [12], Wang obtained an explicit expression for sums of products of $l$ Bernoulli polynomials and $n-l$ Euler polynomials.

In [3], Carlitz also defined the degenerate Euler numbers $\varepsilon_{n}(\lambda)$ by

$$
\frac{2}{(1+\lambda t)^{1 / \lambda}+(1+\lambda t)^{-1 / \lambda}}=\sum_{n=0}^{\infty} \varepsilon_{n}(\lambda) \frac{t^{n}}{n !}
$$

Motivated by (1.4), we shall establish a generalization of (1.5) for $\varepsilon_{n}(\lambda)$ in this note. We define a class of generalized Stirling-like polynomials of the first kind as follows:

$$
\tau_{m, k}(\lambda, x, n)=\sum_{1 \leq i_{k}<i_{k-1}<\cdots<i_{2}<i_{1} \leq m} \prod_{j=1}^{k}\left(x+(\lambda-1) i_{j}-(n+m+1-j) \lambda\right) .
$$

In particular, here we set $\tau_{m, 0}(\lambda, x, n)=1$.

Theorem 1.1 For $1 \leq N \leq n$, we have

$$
\begin{aligned}
& \sum_{\substack{j_{1}+j_{2}+\cdots+j_{N}=n \\
j_{1}, j_{2}, \ldots, j_{N} \geq 0}}\left(\begin{array}{c}
n \\
j_{1}, j_{2}, \ldots, j_{N}
\end{array}\right) \varepsilon_{j_{1}}(\lambda) \varepsilon_{j_{2}}(\lambda) \cdots \varepsilon_{j_{N}}(\lambda) \\
& =\frac{2^{n+N-1}}{(N-1) !} \sum_{k=0}^{N-1}(-1)^{k} \tau_{N-1, k}\left(\frac{\lambda}{2}, \frac{N}{2}, n\right) \varepsilon_{n+N-1-k}\left(\frac{\lambda}{2}, \frac{N}{2}\right),
\end{aligned}
$$

where the degenerate Euler polynomials $\varepsilon_{n}(\lambda, x)$ are given by

$$
\frac{2(1+\lambda t)^{x / \lambda}}{(1+\lambda t)^{1 / \lambda}+1}=\sum_{n=0}^{\infty} \varepsilon_{n}(\lambda, x) \frac{t^{n}}{n !}
$$

In fact, we shall prove a polynomial extension of (1.6) in the next section. In the third section, we also establish a generalization of (1.4) for the degenerate Bernoulli polynomial $\beta_{n}(\lambda, x)$ (see, e.g., [3]).

\section{Degenerate Euler numbers and polynomials}

Note that $\varepsilon_{n}(\lambda)=2^{n} \varepsilon_{n}(\lambda / 2,1 / 2)$. So (1.6) is evidently a consequence of the following theorem.

Theorem 2.1 Let $y=x_{1}+x_{2}+\cdots+x_{N}$. Then for $1 \leq N \leq n$, we have

$$
\begin{aligned}
& \sum_{\substack{j_{1}+j_{2}+\cdots+j_{N}=n \\
j_{1}, j_{2}, \ldots, j_{N} \geq 0}}\left(\begin{array}{c}
n \\
j_{1}, j_{2}, \ldots, j_{N}
\end{array}\right) \varepsilon_{j_{1}}\left(\lambda, x_{1}\right) \varepsilon_{j_{2}}\left(\lambda, x_{2}\right) \cdots \varepsilon_{j_{N}}\left(\lambda, x_{N}\right) \\
& =\frac{2^{N-1}}{(N-1) !} \sum_{k=0}^{N-1}(-1)^{k} \tau_{N-1, k}(\lambda, y, n) \varepsilon_{n+N-1-k}(\lambda, y) .
\end{aligned}
$$


The degenerate Euler polynomials of order $m$ are defined by

$$
\left(\frac{2}{(1+\lambda t)^{1 / \lambda}+1}\right)^{m}(1+\lambda t)^{x / \lambda}=\sum_{n=0}^{\infty} \varepsilon_{n}^{(m)}(\lambda, x) \frac{t^{n}}{n !} .
$$

Clearly, $\varepsilon_{n}^{(1)}(\lambda, x)=\varepsilon_{n}(\lambda, x)$.

Lemma 2.1 For $1 \leq m \leq n$, we have

$$
\varepsilon_{n}^{(m)}(\lambda, x)=\frac{2^{m-1}}{(m-1) !} \sum_{k=0}^{m-1}(-1)^{k} \tau_{m-1, k}(\lambda, x, n) \varepsilon_{n+m-1-k}(\lambda, x) .
$$

Proof Observe that

$$
\begin{aligned}
& \frac{2^{m+1}(1+\lambda t)^{x / \lambda}}{\left((1+\lambda t)^{1 / \lambda}+1\right)^{m+1}} \\
& =\frac{2(1+\lambda t)}{m} \cdot \frac{d}{d t}\left(\frac{2^{m}(1+\lambda t)^{x / \lambda}}{\left((1+\lambda t)^{1 / \lambda}+1\right)^{m}}\right) \\
& \quad-\frac{2(x-m)}{m} \cdot \frac{2^{m}(1+\lambda t)^{x / \lambda}}{\left((1+\lambda t)^{1 / \lambda}+1\right)^{m}} .
\end{aligned}
$$

Comparing the coefficients of $t^{n}$ in both sides of the above equation, we have

$$
\varepsilon_{n}^{(m+1)}(\lambda, x)=\frac{2}{m}\left(\varepsilon_{n+1}^{(m)}(\lambda, x)-(x+(\lambda-1) m-(n+m) \lambda) \varepsilon_{n}^{(m)}(\lambda, x)\right) .
$$

Below we use induction on $m$ to show (2.3). It is easy to see that (2.3) holds for $m=1$. Now let $m>1$ and assume that (2.3) holds for the smaller values of $m$. Then, by the induction hypothesis, we have

$$
\begin{aligned}
\frac{2}{m} \cdot \varepsilon_{n+1}^{(m)}(\lambda, x)-\frac{2}{m} \cdot(x+(\lambda-1) m-(n+m) \lambda) \varepsilon_{n}^{(m)}(\lambda, x) \\
=\frac{2^{m}}{m !} \sum_{k=0}^{m-1}(-1)^{k} \tau_{m-1, k}(\lambda, x, n+1) \varepsilon_{n+m-k}(\lambda, x) \\
\quad-\frac{2^{m}(x+(\lambda-1) m-(n+m) \lambda)}{m !} \sum_{k=1}^{m}(-1)^{k-1} \tau_{m-1, k-1}(\lambda, x, n) \varepsilon_{n+m-k}(\lambda, x) .
\end{aligned}
$$

It is easy to verify that

$$
\tau_{m, k}(\lambda, x, n)=\tau_{m-1, k}(\lambda, x, n+1)+(x+(\lambda-1) m-(n+m) \lambda) \tau_{m-1, k-1}(\lambda, x, n)
$$

for $1 \leq k \leq m-1$, and

$$
\tau_{m, m}(\lambda, x, n)=(x+(\lambda-1) m-(n+m) \lambda) \tau_{m-1, m-1}(\lambda, x, n) .
$$


So by (2.4), we get

$$
\begin{aligned}
& \varepsilon_{n}^{(m+1)}(\lambda, x) \\
& =\frac{2^{m}}{m !}\left(\varepsilon_{n+m}(\lambda, x)+(-1)^{m} \tau_{m, m}(\lambda, x, n) \varepsilon_{n}(\lambda, x)+\sum_{k=1}^{m-1}(-1)^{k} \tau_{m, k}(\lambda, x, n) \varepsilon_{n+m-k}(\lambda, x)\right) \\
& =\frac{2^{m}}{m !} \sum_{k=0}^{m}(-1)^{k} \tau_{m, k}(\lambda, x, n) \varepsilon_{n+m-k}(\lambda, x) .
\end{aligned}
$$

This concludes our proof.

Let us turn to the proof of Theorem 2.1. Clearly,

$$
\begin{aligned}
& \frac{2(1+\lambda t)^{x_{1} / \lambda}}{(1+\lambda t)^{1 / \lambda}+1} \cdot \frac{2(1+\lambda t)^{x_{2} / \lambda}}{(1+\lambda t)^{1 / \lambda}+1} \cdots \frac{2(1+\lambda t)^{x_{N} / \lambda}}{(1+\lambda t)^{1 / \lambda}+1} \\
& =\frac{2^{N}(1+\lambda t)^{\left(x_{1}+x_{2}+\cdots+x_{N}\right) / \lambda}}{\left((1+\lambda t)^{1 / \lambda}+1\right)^{N}} .
\end{aligned}
$$

Hence we have

$$
\sum_{\substack{j_{1}+j_{2}+\cdots+j_{N}=n \\
j_{1}, j_{2}, \ldots, j_{N} \geq 0}}\left(\begin{array}{c}
n \\
j_{1}, j_{2}, \ldots, j_{N}
\end{array}\right) \varepsilon_{j_{1}}\left(\lambda, x_{1}\right) \varepsilon_{j_{2}}\left(\lambda, x_{2}\right) \cdots \varepsilon_{j_{N}}\left(\lambda, x_{N}\right)=\varepsilon_{n}^{(N)}(\lambda, y) .
$$

Thus (2.1) immediately follows from (2.3).

\section{Degenerate Bernoulli numbers and polynomials}

The Bernoulli polynomials $B_{n}(x)$ are defined by

$$
\frac{t e^{x t}}{e^{t}-1}=\sum_{n=0}^{\infty} B_{n}(x) \frac{t^{n}}{n !}
$$

Clearly, $B_{n}(0)=B_{n}$. In [1], Dilcher proved that

$$
\begin{aligned}
& \sum_{\substack{j_{1}+j_{2}+\cdots+j_{N}=n \\
j_{1}, j_{2}, \ldots, j_{N} \geq 0}}\left(\begin{array}{c}
n \\
j_{1}, j_{2}, \ldots, j_{N}
\end{array}\right) B_{j_{1}}\left(x_{1}\right) B_{j_{2}}\left(x_{2}\right) \cdots B_{j_{N}}\left(x_{N}\right) \\
& =N\left(\begin{array}{c}
n \\
N
\end{array}\right) \sum_{j=0}^{N-1}(-1)^{N-1-j}\left(\sum_{k=0}^{j}\left(\begin{array}{c}
n-1-k \\
j-k
\end{array}\right) s(N, N-k) y^{j-k}\right) \cdot \frac{B_{n-j}(y)}{n-j},
\end{aligned}
$$

where $y=x_{1}+x_{2}+\cdots+x_{N}$.

The degenerate Bernoulli polynomials $\beta_{n}(\lambda, x)$ are defined by

$$
\frac{t(1+\lambda t)^{x / \lambda}}{(1+\lambda t)^{1 / \lambda}-1}=\sum_{n=0}^{\infty} \beta_{n}(\lambda, x) \frac{t^{n}}{n !}
$$

In this section, we shall give a generalization of (3.1) for $\beta_{n}(\lambda, x)$. 
Define $\sigma_{m, k}(\lambda, x, n)$ by

$$
\sigma_{m, k}(\lambda, x, n)=\sum_{1 \leq i_{k}<i_{k-1}<\cdots<i_{2}<i_{1} \leq m} \prod_{j=1}^{k}\left(x+(\lambda-1) i_{j}-(n-j) \lambda\right) .
$$

In particular, we set $\sigma_{m, 0}(\lambda, x, n)=1$.

Theorem 3.1 Let $y=x_{1}+x_{2}+\cdots+x_{N}$. Then for $1 \leq N \leq n$, we have

$$
\begin{gathered}
\sum_{\substack{j_{1}+j_{2}+\cdots+j_{N}=n \\
j_{1}, j_{2}, \ldots, j_{N} \geq 0}}\left(\begin{array}{c}
n \\
j_{1}, j_{2}, \ldots, j_{N}
\end{array}\right) \beta_{j_{1}}\left(\lambda, x_{1}\right) \beta_{j_{2}}\left(\lambda, x_{2}\right) \cdots \beta_{j_{N}}\left(\lambda, x_{N}\right) \\
=N\left(\begin{array}{c}
n \\
N
\end{array}\right) \sum_{k=0}^{N-1}(-1)^{N-1-k} \sigma_{N-1, k}(\lambda, y, n) \cdot \frac{\beta_{n-k}(\lambda, y)}{n-k} .
\end{gathered}
$$

Proof Similarly as in the proof of Theorem 2.1, it suffices to show that

$$
\beta_{n}^{(m)}(\lambda, x)=m\left(\begin{array}{l}
n \\
m
\end{array}\right) \sum_{k=0}^{m-1}(-1)^{m-1-k} \sigma_{m-1, k}(\lambda, x, n) \cdot \frac{\beta_{n-k}(\lambda, x)}{n-k}
$$

for $1 \leq m \leq n$, where the degenerate Bernoulli polynomials of order $m$ are defined by

$$
\left(\frac{t}{(1+\lambda t)^{1 / \lambda}-1}\right)^{m}(1+\lambda t)^{x / \lambda}=\sum_{n=0}^{\infty} \beta_{n}^{(m)}(\lambda, x) \frac{t^{n}}{n !}
$$

We shall use induction on $m$. Clearly (3.4) holds for $m=1$. Let $m \geq 1$ and assume that (3.4) holds for $m$. Note that

$$
\begin{aligned}
\frac{t^{m+1}(1+\lambda t)^{x / \lambda}}{\left((1+\lambda t)^{1 / \lambda}-1\right)^{m+1}}= & \left((1+\lambda t)+(x-m) \cdot \frac{t}{m}\right) \cdot \frac{t^{m}(1+\lambda t)^{x / \lambda}}{\left((1+\lambda t)^{1 / \lambda}-1\right)^{m}} \\
& -(1+\lambda t) \cdot \frac{t}{m} \cdot \frac{d}{d t}\left(\frac{t^{m}(1+\lambda t)^{x / \lambda}}{\left((1+\lambda t)^{1 / \lambda}-1\right)^{m}}\right) .
\end{aligned}
$$

Comparing the coefficients of $\frac{t^{n}}{n !}$ in both sides of the above equation, we get

$$
\beta_{n}^{(m+1)}(\lambda, x)=\frac{m-n}{m} \cdot \beta_{n}^{(m)}(\lambda, x)+\frac{n}{m} \cdot(x+(\lambda-1) m-(n-1) \lambda) \beta_{n-1}^{(m)}(\lambda, x) .
$$

Applying (3.4) for $\beta_{n}^{(m)}(\lambda, x)$ and $\beta_{n-1}^{(m)}(\lambda, x)$, we have

$$
\begin{aligned}
& \frac{m-n}{m} \cdot \beta_{n}^{(m)}(\lambda, x)+\frac{n}{m} \cdot(x+(\lambda-1) m-(n-1) \lambda) \cdot \frac{\beta_{n-1}^{(m)}(\lambda, x)}{n-1} \\
& \quad=(m+1)\left(\begin{array}{c}
n \\
m+1
\end{array}\right)\left(-\sum_{k=0}^{m-1}(-1)^{m-1-k} \sigma_{m-1, k}(\lambda, x, n) \cdot \frac{\beta_{n-k}(\lambda, x)}{n-k}\right. \\
& \left.\quad+(x+(\lambda-1) m-(n-1) \lambda) \sum_{k=1}^{m}(-1)^{m-k} \sigma_{m-1, k-1}(\lambda, x, n-1) \cdot \frac{\beta_{n-k}(\lambda, x)}{n-k}\right) .
\end{aligned}
$$


It is not difficult to check that

$$
\sigma_{m, k}(\lambda, x, n)=\sigma_{m-1, k}(\lambda, x, n)+(x+(\lambda-1) m-(n-1) \lambda) \sigma_{m-1, k-1}(\lambda, x, n-1)
$$

for $1 \leq k \leq m-1$, and

$$
\sigma_{m, m}(\lambda, x, n)=(x+(\lambda-1) m-(n-1) \lambda) \sigma_{m-1, m-1}(\lambda, x, n-1) .
$$

It follows from (3.6) that

$$
\begin{aligned}
\beta_{n}^{(m+1)}(\lambda, x)= & (m+1)\left(\begin{array}{c}
n \\
m+1
\end{array}\right)\left((-1)^{m} \frac{\beta_{n}(\lambda, x)}{n}+\sigma_{m, m}(\lambda, x, n) \frac{\beta_{n-m}(\lambda, x)}{n-m}\right) \\
& +(m+1)\left(\begin{array}{c}
n \\
m+1
\end{array}\right) \sum_{k=1}^{m-1}(-1)^{m-k} \sigma_{m, k}(\lambda, x, n) \frac{\beta_{n-k}(\lambda, x)}{n-k} \\
= & (m+1)\left(\begin{array}{c}
n \\
m+1
\end{array}\right) \sum_{k=0}^{m}(-1)^{m-k} \sigma_{m, k}(\lambda, x, n) \cdot \frac{\beta_{n-k}(\lambda, x)}{n-k} .
\end{aligned}
$$

All proofs thus are done.

\section{Competing interests}

The authors did not provide this information.

\section{Authors' contributions}

The authors did not provide this information.

\section{Author details}

'Department of Basic Courses, JinLing Institute of Technology, Nanjing, 211169, People's Republic of China. ${ }^{2}$ Department of Mathematics, Nanjing University, Nanjing, 210093, People's Republic of China.

\section{Acknowledgements}

The authors are grateful to two anonymous referees for their helpful suggestions. The second author is supported by National Natural Science Foundation of China (Grant No.11271185). The second author is the corresponding author.

\section{Received: 2 October 2013 Accepted: 8 January 2014 Published: 27 Jan 2014}

\section{References}

1. Dilcher, K: Sums of products of Bernoulli numbers. J. Number Theory 60, 23-41 (1996)

2. Comtet, L: Advanced Combinatorics. Reidel, Dordrecht (1974)

3. Carlitz, L: Degenerate Stirling, Bernoulli and Eulerian numbers. Util. Math. 15(1), 51-88 (1979)

4. Carlitz, L: A generate Staudt-Clausen theorem. Arch. Math. 7(1), 28-33 (1956)

5. Howard, FT: Explicit formulas for degenerate Bernoulli numbers. Discrete Math. 162, 175-185 (1996)

6. Shirai, S: Some identities involving Bernoulli and Stirling numbers. J. Number Theory 90, 130-142 (2001)

7. Young, PT: Congruences for degenerate number sequences. Discrete Math. 270, 279-289 (2003)

8. Young, PT: Degenerate Bernoulli polynomials, generalized factorial sums, and their applications. J. Number Theory $128,738-758(2008)$

9. Wu, M, Pan, H: Sums of products of Bernoulli numbers of the second kind. Fibonacci Q. 45, 146-150 (2007)

10. Fu, AM, Zhang, IF: Inverse relations and the products of Bernoulli polynomials. Graphs Comb. 26, 361-367 (2010)

11. Zhang, Z, Yang, J: On sums of products of the degenerate Bernoulli numbers. Integral Transforms Spec. Funct. 20(10), 751-755 (2009)

12. Wang, W: Some results on sums of products of Bernoulli polynomials and Euler polynomials. Ramanujan J. 32 , 159-184 (2013)

10.1186/1687-1847-2014-40

Cite this article as: Wu and Pan: Sums of products of the degenerate Euler numbers. Advances in Difference Equations $2014,2014: 40$ 